USTC-ICTS-16-13

\title{
Virtual states and generalized completeness relation in the Friedrichs Model
}

\author{
Zhiguang Xiad* \\ Interdisciplinary Center for Theoretical Study, University of Science and Technology of China, Hefei, Anhui 230026, China \\ Zhi-Yong Zhou田 \\ Department of Physics, Southeast University, Nanjing 211189, P. R. China and \\ Kavli Institute for Theoretical Physics China, CAS, Beijing 100190, China
}

(Dated: March 5, 2018)

\begin{abstract}
We study the well-known Friedrichs model, in which a discrete state is coupled to a continuum state. By examining the pole behaviors of the Friedrichs model in a specific form factor thoroughly, we find that, in general, when the bare discrete state is below the threshold of the continuum state, there should also be a virtual-state pole accompanying the bound-state pole originating from the bare discrete state as the coupling is turned on. There are also other second-sheet poles originating from the singularities of the form factor. We give a general argument for the existence of these two kinds of states. As the coupling is increased to a certain value, the second-sheet poles may merge and become higher-order poles. We then discuss the completeness relations incorporating bound states, virtual states, and resonant states corresponding to higher-order poles.
\end{abstract}

PACS numbers: 11.10.St, 11.55.Bq, 12.39.Pn, 11.55.Fv

* \begin{tabular}{l} 
xiaozg@ustc.edu.cn \\
\hline zhouzhy@seu.edu.cn
\end{tabular} 


\section{INTRODUCTION}

Unstable states in quantum physics appear in a lot of fields in modern physics, such as unstable nuclei in nuclear physics and resonances in particle physics. In hadron physics especially, unstable resonances always arise in the strong interactions, and more and more newly observed resonant states are quoted in the Particle Data Group Table [1]. However, many states fall outside the expectations of the conventional quark models such as the Godfrey-Isgur model [2], in which mesons are regarded as the bound state of a quark and an antiquark in some potential induced by QCD. Typically, the enigmatic $\sigma$ and $\kappa$ resonances in $\pi \pi$ and $\pi K$ scatterings [3, 4] can hardly be accommodated in the conventional quark model, because they are strongly coupled with the continuum states. In the higher energy region, e.g. for the quarkonium-like states near or above the open-flavor thresholds, such as $X(3872), D_{0}^{*}(2318), D_{s 0}^{*}(2317)$, $X(3900)$, the conventional quark models neither work well. An interesting approach by taking into account the hadron loop effects provides a generally good description to the masses and widths of the resonances ranging from the light scalars [5] to the heavier charmed, charmed-strange, and charmonium-like spectra [6 8]. From the point of view of the hadron-loop model, one may roughly regard these resonances as being composed partly by the discrete spectra and the continuum spectra of the free Hamiltonian without their interaction. How to describe these resonances in terms of these components in a more rigorous way in quantum theory inspires us to look at the mathematical description of the resonances.

The description of resonance, i.e. Gamow states, cannot be formulated in the usual Hilbert space language, since it has complex energy eigenvalue. In order to complete the task, one has to enlarge the usual Hilbert space to a rigged Hilbert space (RHS). The main point is to have a Gel'fand triplet $\Omega \subset \mathscr{H} \subset \Omega^{\times}$, where $\mathscr{H}$ is the usual Hilbert space of the normalizable states, $\Omega$ is a nuclear space which is dense in $\mathscr{H}$, and $\Omega^{\times}$is the space of the antilinear continuous functionals on the nuclear space. Gamow states must be in the larger $\Omega^{\times}$, since it is the generalized eigenstate of the full Hamiltonian with complex eigenvalues. The descriptions of in-state and out-state are using different rigged Hilbert spaces, $\Omega_{ \pm} \subset \mathscr{H} \subset \Omega_{ \pm}^{\times}$where the subscript" - " denotes the out-state space and + denotes the in-state space. The triplet of state spaces can be mapped to the complex function spaces $D_{\mp} \subset \mathscr{H}_{\mp}^{2} \subset D_{\mp}^{\times}$where $D_{\mp}=\left.S \cap \mathscr{H}_{\mp}^{2}\right|_{\mathbb{R}^{+}}$, respectively, to form a representation, where $S$ is the Schwartz space, $\mathscr{H}_{\mp}^{2}$ is the so-called Hardy space in which the functions are analytic on $\mathbb{C}_{\mp}$, and $\left.\right|_{\mathbb{R}^{+}}$means restriction on $\mathbb{R}^{+}$. There are also two kinds of Gamow states, $\left|z_{R}^{-}\right\rangle \in \Omega_{-}^{\times}$, $\left|z_{R}^{+}\right\rangle \in \Omega_{+}^{\times}$denoting the decaying state and growing states, which correspond to the lower and upper second-sheet poles of the $S$ matrix, respectively. For further detailed discussion on the mathematical foundation, the readers are referred to $[9,10]$.

The Friedrichs model [11] is a solvable model which demonstrates the property of the Gamow state. In the simplest Friedrichs model [1] there are a discrete eigenstate and a continuum eigenstate of the free Hamiltonian, which couple to each other through an interaction term in the full Hamiltonian. The eigenstates of the full Hamiltonian can be worked out exactly. If the free discrete state is located above the threshold of the continuum, it will become unstable Gamow states represented as a pair of poles on the second Riemann sheet of the $S$ matrix. The pole on the lower second sheet corresponds to the decaying Gamow state and the one on the upper corresponds to the growing state. The Gamow state can be explicitly written down as a vector in the RHS as a linear combination of the free discrete state and the continuum states. Besides these resonance poles generated from the discrete states, there could also be other poles introduced by the form factors. This was noticed in Ref. 12] by studying some special form factors and the authors argue that it is a possible origin of extra states in particle physics. In present paper, we will give a general arguement of the existence of these states introduced by the form factors. If the free discrete state is below the threshold of the continuum, there is a bound-state pole on the physical sheet below the threshold after turning on the interaction. However, people usually do not notice that, besides this bound-state pole, there will also be a virtual state on the unphysical sheet originating from the same discrete state. This virtual state pole is the so-called shadow pole proposed by Eden [13] in $S$-matrix theory. In our present paper, we will demonstrate the existence of this virtual-state pole in the Friedrichs model by studying some example form factors and give a general argument of its existence. We also track the pole trajectories as the couplings change continuously and we find that even for such a simple form factor there are unexpected pole structures. The pair of the resonance poles can merge to be a degenerate double pole and then separate on the real axis, becoming two virtual-state poles. One of these virtual poles may meet with the virtual pole generated by the form factor and then they separate into the complex plane becoming a pair of resonance poles. In some special critical condition, the pair of resonance poles and the virtual pole originating from the form factor may meet on the negative real axis and form a triple pole. The double pole can be regarded as two degenerate states and the triple pole as three degenerate ones. In fact, the higher order degenerate states are not the eigenstates of the Hamiltonian. In the bases of these degenerate states, the Hamiltonian is represented as the matrix of Jordan form. One would wonder whether these states would contribute to the completeness relations. In fact, for nondegenerate Gamow states for the resonances, by suitably incorporating the integral contour information in the continuum state as a distribution, the identity operator can be decomposed in the continuum states and the Gamow 
states [14]. Here we will generalize the completeness relations to the degenerate higher order states in the Friedrichs model.

The structure of this paper is as follows: Section @introduces the Friedrichs model. In Sec. III we examine pole trajectories of the Friedrichs model with a kind of example form factor. In Sec. IV we give a general argument for the existence of the virtual state accompanying the bound state and the states generated by form factor. In Sec. $\mathbb{V}$. we study the completeness relations with higher-order degenerate states. Section $\nabla 1$ is the conclusion and discussion.

\section{INTRODUCTION TO THE FRIEDRICHS MODEL}

The simplest Friedrichs model [11] includes a free Hamiltonian $H_{0}$ with a simple continuous spectrum, which is $\mathbb{R}^{+} \equiv[0, \infty)$, plus a discrete eigenvalue $\omega_{0}$ imbedded in this continuous spectrum $\left(\omega_{0}>0\right)$. An interaction $V$ between the continuous and discrete parts is produced so that the discrete state of $H_{0}$ is dissolved in the continuous spectrum and a resonance is produced. In fact, all the following solutions also apply to $\omega_{0}<0$ cases, so we would not restrict the domain of $\omega_{0}$ for the moment. We denote the discrete state of $H_{0}$ by $|1\rangle$ and the continuum state by the $|\omega\rangle$, that is,

$$
\begin{gathered}
H_{0}|1\rangle=\omega_{0}|1\rangle, \\
H_{0}|\omega\rangle=\omega|\omega\rangle .
\end{gathered}
$$

The free Hamiltonian is then

$$
H_{0}=\omega_{0}|1\rangle\left\langle 1\left|+\int_{0}^{\infty} \omega\right| \omega\right\rangle\langle\omega| \mathrm{d} \omega
$$

and the interaction $V$ is written as

$$
V=\lambda \int_{0}^{\infty}[f(\omega)|\omega\rangle\langle 1|+f(\omega)| 1\rangle\langle\omega|] \mathrm{d} \omega .
$$

For simplification, we assume that $f(\omega)$ is real for above the threshold, i.e. $\omega>0$. In general, it could be a complex function. The normalizations and orthogonal conditions for the free states are

$$
\langle 1 \mid 1\rangle=1,\langle 1 \mid \omega\rangle=\langle\omega \mid 1\rangle=0,\left\langle\omega \mid \omega^{\prime}\right\rangle=\left\langle\omega^{\prime} \mid \omega\right\rangle=\delta\left(\omega-\omega^{\prime}\right) .
$$

We will solve the eigenstate $|\Psi(x)\rangle$ of $H=H_{0}+V$ with eigenvalue $x$,

$$
H \Psi(x)=x|\Psi(x)\rangle .
$$

Since $|1\rangle$ and $|\omega\rangle$ form a complete set, the eigenstate $|\Psi(x)\rangle$ can be expressed in terms of $|1\rangle$ and $|\omega\rangle$,

$$
|\Psi(x)\rangle=\alpha(x)|1\rangle+\int_{0}^{\infty} \psi(x, \omega)|\omega\rangle \mathrm{d} \omega .
$$

Note that here $|\Psi\rangle$ is a vector in $\Phi^{\times}$, and it only make senses as an antilinear functional on the vector $|\phi\rangle \in \Phi,\langle\phi \mid \Psi\rangle$. So, $\psi(x, \omega)$ should be treated as a distribution. Substituting (6) into Eq.(5), one can obtain the following relations:

$$
\begin{array}{r}
\left(\omega_{0}-x\right) \alpha(x)+\lambda \int_{0}^{\infty} f(\omega) \psi(x, \omega) \mathrm{d} \omega=0 \\
(\omega-x) \psi(x, \omega)+\lambda f(\omega) \alpha(x)=0 .
\end{array}
$$

Then, for real $x>0$, we have

$$
\begin{gathered}
\psi_{ \pm}(x, \omega)=-\frac{\lambda \alpha(x) f(\omega)}{\omega-x \pm i \epsilon}+\gamma(\omega) \delta(\omega-x) \\
\left(\omega_{0}-x\right) \alpha_{ \pm}(x)+\lambda f(x) \gamma(x)-\alpha_{ \pm}(x) \lambda^{2} \int_{0}^{\infty} \frac{|f(\omega)|^{2}}{\omega-x \pm i \epsilon} \mathrm{d} \omega=0 .
\end{gathered}
$$

where $\gamma(\omega)$ is an arbitrary function to be determined by normalization. We have added the $\pm i \epsilon$ in order to define the integral contour. Now, one can define

$$
\eta^{ \pm}(x)=x-\omega_{0}-\lambda^{2} \int_{0}^{\infty} \frac{|f(\omega)|^{2}}{x-\omega \pm i \epsilon} \mathrm{d} \omega,
$$




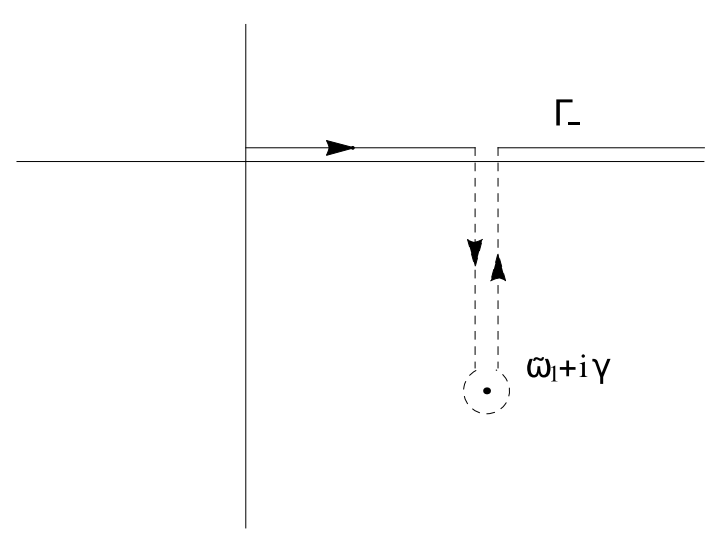

FIG. 1. The deformation of the integral path.

and analytically continue $\eta^{ \pm}$to the complex plane $\eta(x)$, and $\eta^{+}$and $\eta^{-}$are the boundary functions of $\eta(x)$ on the upper rim and lower rim of the cut on the positive axis, respectively. Since $\eta(x)$ is a real-analytic function, satisfying the Schwartz reflection relation $\eta^{*}(z)=\eta\left(z^{*}\right)$, the analytically continued form factor $G(x) \equiv|f(x)|^{2}$, being proportional to the imaginary part of $\eta(x)$, should be anti-real-analytic, that is, $G\left(x^{*}\right)=-G(x)^{*}$. In usual physical applications, $\eta(x)$ is real below the threshold and complex with a positive imaginary part above the threshold. This requires $G(x)$ to positive real function above the threshold and imaginary below the threshold.

After choosing the normalization such that $\left\langle\Psi_{ \pm}\left(x^{\prime}\right) \mid \Psi_{ \pm}(x)\right\rangle=\delta\left(x^{\prime}-x\right)$, one obtains the solution

$$
\left|\Psi_{ \pm}(x)\right\rangle=|x\rangle+\lambda \frac{f(x)}{\eta^{ \pm}(x)}\left[|1\rangle+\lambda \int_{0}^{\infty} \mathrm{d} \omega \frac{f(\omega)}{x-\omega \pm i \epsilon}|\omega\rangle\right]
$$

The two sets of states $\left|\Psi_{+}(x)\right\rangle$ and $\left|\Psi_{-}(x)\right\rangle$ can be understood as in-states and out-states, i.e. $\left|\Psi_{\mp}(x)\right\rangle \in \Omega_{\mp}^{\times}$.

For complex eigenvalue $x$ not on the positive real axis, Eq. (8) would not have the $\gamma(\omega)$ term, and there is no need for $i \epsilon$ in the denominator. In order for $\alpha(x)$ to be nonzero, the eigenvalue must satisfy $\eta(x)=0$. Depending on the positions of the solutions, the eigenstates may be categorized into resonant states, bound states, or virtual states.

1. Resonant states. If $\eta(x)=0$ has a pair of complex conjugate solutions $z_{R} \in \mathbb{C}_{-}$and $z_{R}^{*} \in \mathbb{C}_{+}$on the second sheet, the right eigenstates for eigenvalue $z_{R}$ and $z_{R}^{*}$ can be expressed as

$$
\begin{aligned}
& \left|z_{R}\right\rangle=N_{R}\left(|1\rangle+\lambda \int_{0}^{\infty} \mathrm{d} \omega \frac{f(\omega)}{\left[z_{R}-\omega\right]_{+}}|\omega\rangle\right), \\
& \left|z_{R}^{*}\right\rangle=N_{R}^{*}\left(|1\rangle+\lambda \int_{0}^{\infty} \mathrm{d} \omega \frac{f(\omega)}{\left[z_{R}^{*}-\omega\right]_{-}}|\omega\rangle\right),
\end{aligned}
$$

which are the Gamow states satisfying $H\left|z_{R}\right\rangle=z_{R}\left|z_{R}\right\rangle$ and $H\left|z_{R}^{*}\right\rangle=z_{R}^{*}\left|z_{R}^{*}\right\rangle$. [... $]_{ \pm}$denotes the continuation from the upper rim of the cut to the lower second sheet for "+" or from the lower rim to the upper second sheet for "-", and hence the deformation of the integration path as in Fig. 1 for "+" and the opposite for "-" is needed.

We have also the left eigenstates, i.e. $\left\langle\tilde{z}_{R}\right| H=z_{R}\left\langle\tilde{z}_{R}\right|$ and $\left\langle\tilde{z}_{R}^{*}\right| H=z_{R}^{*}\left\langle\tilde{z}_{R}^{*}\right|$,

$$
\begin{aligned}
& \left\langle\tilde{z}_{R}\right|=N_{R}\left(\langle 1|+\lambda \int_{0}^{\infty} \mathrm{d} \omega \frac{f(\omega)}{\left[z_{R}-\omega\right]_{+}}\langle\omega|\right), \\
& \left\langle\tilde{z}_{R}^{*}\right|=N_{R}^{*}\left(\langle 1|+\lambda \int_{0}^{\infty} \mathrm{d} \omega \frac{f(\omega)}{\left[z_{R}^{*}-\omega\right]_{-}}\langle\omega|\right) .
\end{aligned}
$$

The normalization is chosen as $N_{R}=\left(\eta^{\prime+}\left(z_{R}\right)\right)^{-1 / 2}=\left(1+\lambda^{2} \int d \omega \frac{|f(\omega)|^{2}}{\left[\left(z_{R}-\omega\right)_{+}\right]^{2}}\right)^{-1 / 2}$ such that $\left\langle\tilde{z}_{R} \mid z_{R}\right\rangle=1$, since the resonant state has zero norm $\left\langle z_{R} \mid z_{R}\right\rangle=0$ [14, 15]. 
2. Bound states. If $\eta(x)=0$ have a solution on the negative real axis on physical Riemann sheet, it represents a bound state. The bound state with eigenvalue $z_{B}$ can then be represented as [16, 17]

$$
\left|z_{B}\right\rangle=N_{B}\left(|1\rangle+\lambda \int_{0}^{\infty} \frac{f(\omega)}{z_{B}-\omega}|\omega\rangle \mathrm{d} \omega\right)
$$

where $N_{B}=\left(\eta^{\prime}\left(z_{B}\right)\right)^{-1 / 2}=\left(1+\lambda^{2} \int d \omega \frac{|f(\omega)|^{2}}{\left(z_{B}-\omega\right)^{2}}\right)^{-1 / 2}$.

3. Virtual states. If $\eta(x)=0$ has a solution on the negative real axis of the second Riemann sheet, it corresponds to a virtual state. The general discussion of the virtual states in the rigged Hilbert space formulation can be found in Refs. [18] and [19]. For the simple virtual poles, similar to resonant states, there are two kinds of states, $\left|z_{v}^{+}\right\rangle$by analytical continuation from the upper rim and $\left|z_{v}^{-}\right\rangle$lower rim of the cut to the second sheet

$$
\left|z_{v}^{ \pm}\right\rangle=N_{v}^{ \pm}\left(|1\rangle+\lambda \int_{0}^{\infty} \frac{f(\omega)}{\left[z_{v}-\omega\right]_{ \pm}}|\omega\rangle \mathrm{d} \omega\right), \quad\left\langle\tilde{z}_{v}^{ \pm}\right|=\left\langle z_{v}^{\mp}\right|,
$$

where $N_{v}^{-}=N_{v}^{+*}=\left(\eta^{+}\left(z_{v}\right)\right)^{-1 / 2}=\left(1+\lambda^{2} \int d \omega \frac{|f(\omega)|^{2}}{\left[\left(z_{v}-\omega\right)_{+}\right]^{2}}\right)^{-1 / 2}$. As discussed in Refs. [18] and [19], $\left|z_{v}^{-}\right\rangle$is only defined for $t>0$ representing the out-state and $\left|z_{v}^{+}\right\rangle$for $t<0$ representing the in-state. In some accidental cases, there could be multiple zeroes for $\eta(x)$ on the negative real axis of the unphysical sheet, corresponding to the degenerate virtual states, which will be discussed later.

\section{POLE TRAJECTORIES IN THE FRIEDICHS MODEL WITH AN EXAMPLE FORM FACTOR}

In this section, we will use an example form factor to exhibit the existence of these three kinds of states, and they can transform to each other as the coupling changes.

As a simple integrable example, similar to [12], we choose $|f(\omega)|^{2}=\frac{\sqrt{\omega}}{\omega+\rho^{2}}, \rho>0$ and analyze the pole structures for different parameters. The integral in $\eta(\omega)$ can be worked out and analytically continued to the complex plane,

$$
\eta(\omega)=\omega-\omega_{0}+\frac{\pi \lambda^{2}}{\sqrt{-\omega}+\rho}=\omega-\omega_{0}+\frac{\pi \lambda^{2}}{-i \sqrt{\omega}+\rho} .
$$

Choosing $-i$ for $\sqrt{-1}=\left(e^{-i \pi}\right)^{1 / 2}$ in the denominator makes the imaginary part of $\eta^{+}(x)$ positive at $x>0$. The cut for $\omega$ is along the positive real axis. Continued to the second sheet, $\eta$ reads

$$
\eta^{I I}(\omega)=\omega-\omega_{0}+\frac{\pi \lambda^{2}}{-\sqrt{-\omega}+\rho}
$$

where the superscript $I I$ denotes the second Riemann sheet.

For future convenience, we turn to the momentum plane by making the change of the variable $\omega=u^{2}$. The $u$ plane combines the first sheet and the second sheet of the $\omega$ plane. The first sheet of $\omega$ corresponds to the upper half-plane of $u$, and the second sheet of $\omega$ corresponds to the lower half. The equation $\eta\left(u^{2}\right)=0$ can be recast into

$$
\frac{1}{u+i \rho}\left(u^{3}+i \rho u^{2}-\omega_{0} u-i\left(\rho \omega_{0}-\pi \lambda^{2}\right)\right)=0
$$

There are three solutions for this third-order algebraic equation.

Case 1. We first look at the case for $\omega_{0}>0$ and turning on the coupling constant slowly such that $\omega_{0}>\frac{\pi \lambda^{2}}{\rho}$. This case is studied in [12] and we give a review here for completeness and discuss more on the virtual states from the form factor. In this case, there is no bound state. The three solutions correspond to a pair of resonant states and a virtual state. If we set the three solutions to be $u_{1,2}= \pm \alpha-i \gamma$ and $u_{3}=-i d$, the three solutions can be expanded in orders of $\lambda$,

$$
\begin{aligned}
& E_{1,2}=\omega_{0}-2 d \gamma \mp 2 i \gamma \omega_{0}^{1 / 2}+O\left(\lambda^{4}\right)=\omega_{0}-\frac{\pi \lambda^{2}}{\rho \mp i \omega_{0}^{1 / 2}}+O\left(\lambda^{4}\right), \\
& E_{3}=-(\rho-2 \gamma)^{2}=-\rho^{2}+4 \gamma \rho+O\left(\lambda^{4}\right)=-\rho^{2}+\frac{2 \rho \pi \lambda^{2}}{\omega_{0}+\rho^{2}}+O\left(\lambda^{4}\right)
\end{aligned}
$$


The virtual-state pole at $E_{3}$ does not exist at exact $\lambda=0$, but as long as $\lambda$ runs away from 0 , the residue of the pole is not zero and it appears near the poles of the form factor on the second sheet.

The pair of resonance poles originates from the discrete state can be represented as the Gamow states as in (11). The virtual state can be represented as in (14). One may wonder whether, as $\lambda \rightarrow 0$, the virtual state tends to $|1\rangle$ from (14). This is not correct. In fact, the integral term in (14) is of $\mathcal{O}\left(\lambda^{0}\right)$, since at $\lambda=0$, the integral is divergent. In fact, for the example form factor, for small $\lambda$, the integral term should be of order $\lambda \mathcal{O}(1 / \lambda) \sim \mathcal{O}\left(\lambda^{0}\right)$. To see this, we take the inner product $\left\langle\phi \mid z_{v}^{-}\right\rangle$, where $\left|z_{v}^{-}\right\rangle$is defined in (14) and $|\phi\rangle \in \Phi^{-}$and $\langle\phi \mid \omega\rangle=\left.\phi(\omega) \in S \cap \mathscr{H}_{-}^{2}\right|_{\mathbb{R}^{+}}$. The integral term in the inner product is

$$
\lambda \int_{0}^{\infty} \frac{\omega^{1 / 4} \phi(\omega)}{\left(\omega+\rho^{2}\right)^{1 / 2}\left[z_{v}-\omega\right]_{+}} \mathrm{d} \omega=\lambda \int_{0}^{\infty} \frac{\omega^{1 / 4} \phi(\omega)}{\left(\omega+\rho^{2}\right)^{1 / 2}\left(z_{v}-\omega\right)} \mathrm{d} \omega+2 \pi i \lambda \frac{z_{v}^{1 / 4} \phi\left(z_{v}\right)}{\left(z_{v}+\rho^{2}\right)^{1 / 2}}
$$

The integral term in the first term is not singular and will be $\lambda \cdot \mathcal{O}\left(\lambda^{0}\right) \sim \mathcal{O}\left(\lambda^{1}\right)$. If we take $z_{v}=e^{-i \pi}\left(\rho^{2}+a \lambda^{2}\right)$ on the second sheet, the second term will be

$$
2 \pi i \lambda \frac{z_{v}^{1 / 4} \phi\left(z_{v}\right)}{\left(z_{v}+\rho^{2}\right)^{1 / 2}}=2 \pi i \frac{e^{-i \pi / 4} \rho^{1 / 2} \phi\left(e^{-i \pi} \rho^{2}\right)}{(-a)^{1 / 2}}+O\left(\lambda^{2}\right) \sim \mathcal{O}\left(\lambda^{0}\right)
$$

So, the second term in (14) is of the same order as the first term.

Case 2. For larger $\lambda$ satisfying $0<\omega_{0}<\frac{\pi \lambda^{2}}{\rho}$, there are two cases: the two second-sheet poles may or may not go back to the negative real axis to form virtual states. The critical case separating these two cases is the one in which the three poles come together on the negative axis forming a third-order pole and then get away. Let us examine this critical case first. We set the three degenerate poles to be $u_{1,2,3}=-i d$. One can find out that only when

$$
d=\frac{1}{3} \rho, \quad \omega_{0}=\frac{1}{3} \rho^{2}, \quad \rho=\frac{3}{2}\left(\pi \lambda^{2}\right)^{1 / 3}
$$

can the three poles merge to be a third-order pole, which means the degeneracy of the three states (see Fig. (2). Then as $\lambda$ becomes larger, two of them run into the complex plane to be a pair of resonance poles, and the other remains a virtual-state pole and goes up towards threshold. When $\lambda^{2}>\frac{\rho \omega_{0}}{\pi}$, the virtual state will go through the threshold to the first sheet, becoming a bound state and moving downward towards minus infinity.

According to the discussion in [20], the triple pole can be expressed as three kinds of states: the first is the same as the ordinary virtual state as in (14) and the other two come from the higher-order residues of the continuum state

$$
\begin{aligned}
&\left|z_{v 2}^{ \pm}\right\rangle=-N_{v 2} \lambda \int_{0}^{\infty} \frac{f(\omega)}{\left(\left[z_{v}-\omega\right]_{ \pm}\right)^{2}}|\omega\rangle \mathrm{d} \omega, \quad\left\langle\tilde{z}_{v 2}^{ \pm}\right|=\left\langle z_{v 2}^{\mp}\right|, \\
&\left|z_{v 3}^{ \pm}\right\rangle=N_{v 3} \lambda \int_{0}^{\infty} \frac{f(\omega)}{\left(\left[z_{v}-\omega\right]_{ \pm}\right)^{3}}|\omega\rangle \mathrm{d} \omega, \quad\left\langle\tilde{z}_{v 3}^{ \pm}\right|=\left\langle z_{v 3}^{\mp}\right| .
\end{aligned}
$$

The normalization for the simple virtual state cannot be used here, since $\eta(z)$ has a third-order zero, $\eta^{\prime}\left(z_{v}\right)=0$ and also $\eta^{\prime \prime}\left(z_{v}\right)=0$. We can choose $\left\langle\tilde{z}_{v 3}^{ \pm} \mid z_{v}^{ \pm}\right\rangle=1$ and $\left\langle\tilde{z}_{v 2}^{ \pm} \mid z_{v 2}^{ \pm}\right\rangle=1$. Then $N_{v}=N_{v_{2}}=N_{v 3}=\left(6 / \eta^{\prime \prime \prime}\right)^{1 / 2}$. The Hamiltonian is not diagonalized by these states and can only be represented as Jordan form, that is,

$$
\begin{gathered}
H\left|z_{v 3}^{ \pm}\right\rangle=z_{v}\left|z_{v 3}^{ \pm}\right\rangle+2\left|z_{v 2}^{ \pm}\right\rangle, \\
H\left|z_{v 2}^{ \pm}\right\rangle=z_{v}\left|z_{v 2}^{ \pm}\right\rangle+\left|z_{v}^{ \pm}\right\rangle .
\end{gathered}
$$

If the relation between $\omega_{0}$ and $\rho$ in above condition (20) is not satisfied, one possibility is that the two resonance poles can merge at a point different from the virtual state when $\omega_{0}<\frac{\rho^{2}}{3}$. In this case, we set the three pole positions as $u_{1,2}=-i d$ and $u_{3}=-i d_{0}$, and the solution can then be obtained

$$
d=\frac{1}{3}\left(\rho \pm\left(\rho^{2}-3 \omega_{0}\right)^{1 / 2}\right), \quad d_{0}=\frac{1}{3}\left(\rho \mp 2\left(\rho^{2}-3 \omega_{0}\right)^{1 / 2}\right)
$$

There are two solutions which means there are two points where either the two resonance poles come together to one point on the real axis or separate from one point on the real axis. The whole picture is as follows: The original two resonance poles merge first and then separate on the negative real axis becoming two virtual states. One virtual state moves down and meets the original virtual state and then they separate into the complex plane, becoming a pair of resonance poles. The other virtual state moves through the threshold at the origin to the first sheet and becomes a bound state. The bound state then moves down towards negative infinity (see Fig. 31). 


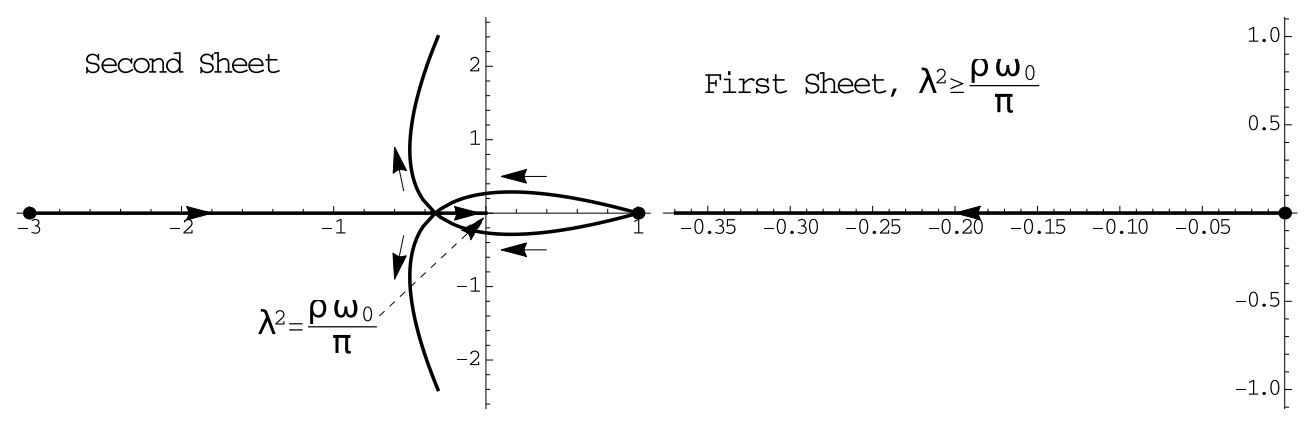

FIG. 2. The pole trajectories as $\lambda$ increases from 0 when $\omega_{0}=\frac{1}{3} \rho^{2} \cdot \rho^{2}=3, \omega_{0}=1$. The three second-sheet poles merge and then separate. Two of them become a pair of resonance poles and the other moves up to first sheet.

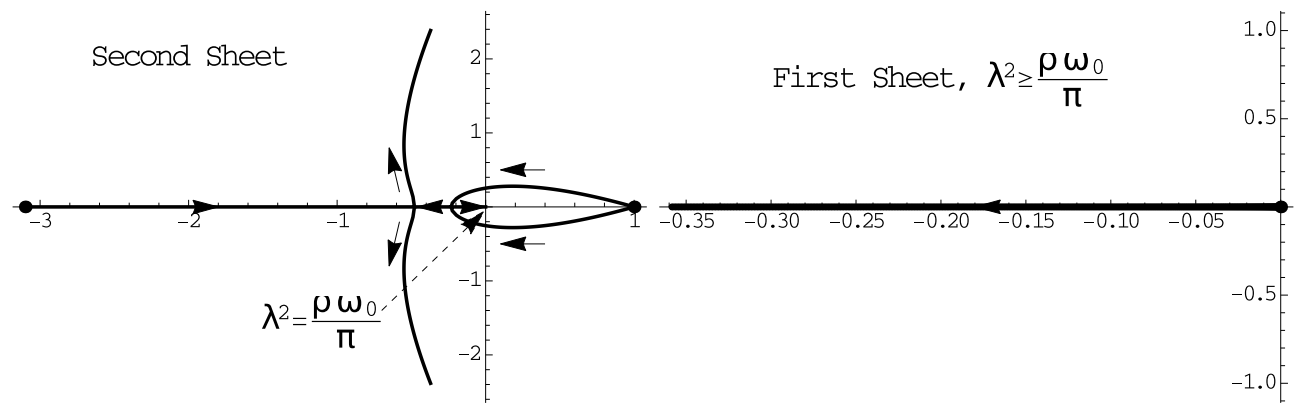

FIG. 3. The pole trajectories as $\lambda$ increases from 0 when $\omega_{0}<\frac{1}{3} \rho^{2}$. We choose $\rho^{2}=3.1, \omega_{0}=1$. The three second sheet poles merge and then separate. Two become a pair of resonance poles and the other virtual state moves up to the first sheet.

Similar to the third-order pole, the second-order pole is a degenerate of two states: the first state is the same as the ordinary virtual state (14), and the second comes from the second-order pole of the continuum state

$$
\left|z_{v 2}^{\mp}\right\rangle=-N_{v 2}^{\mp} \lambda \int_{0}^{\infty} \frac{f(\omega)}{\left(\left[z_{v}-\omega\right]_{ \pm}\right)^{2}}|\omega\rangle \mathrm{d} \omega, \quad\left\langle\tilde{z}_{v 2}^{ \pm}\right|=\left\langle z_{v 2}^{\mp}\right|
$$

The normalizations $N_{v}^{-}=N_{v_{2}}^{-}=\left(2 / \eta^{\prime \prime}\right)^{1 / 2}$ and $N_{v}^{+}=N_{v_{2}}^{+}=N_{v}^{-*}$ are chosen such that $\left\langle\tilde{z}_{v_{2}}^{ \pm} \mid z_{v}^{ \pm}\right\rangle=1$. The secondorder resonance poles were also found in a kind of one-dimensional double barrier potential [21] and in Friedrichs model using another form factor in [22]. Higher-order resonance poles are found to represent the degenerate resonances in [23] and are formulated in RHS language in [20, 24].

The other possibility is that the two resonance poles do not meet and just run away towards infinity. The condition is $\omega_{0}>\rho^{2} / 3$. The virtual state in second sheet just moves to the threshold and comes up to the first sheet, turning into a bound state, and then moves down toward negative infinity ( see Fig. 团).



FIG. 4. The pole trajectories as $\lambda$ increases from 0 when $\omega_{0}>\frac{1}{3} \rho^{2}$. We choose $\rho^{2}=1, \omega_{0}=1$. The three second-sheet poles do not merge. The virtual-state pole moves up to the first sheet for large $\lambda$. 


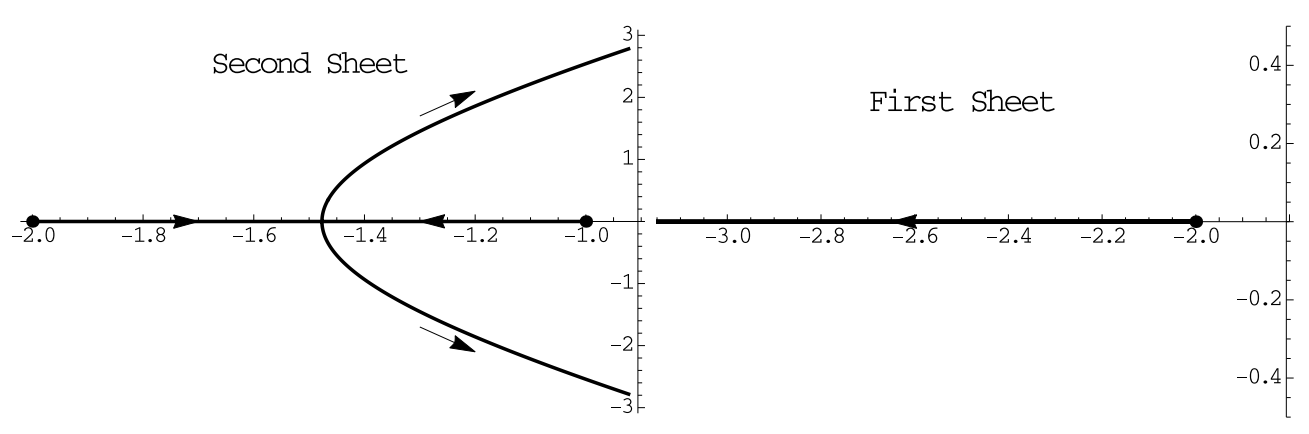

FIG. 5. The pole trajectories as $\lambda$ increases from 0 when $\omega_{0}<0 . \rho^{2}=1, \omega_{0}=-2$. The two virtual poles merge and then separate to a pair of resonance poles. The bound-state pole moves to negative infinity.

Case 3. $\omega_{0}<0$. There is always a bound-state pole on the first Riemann sheet. $\eta=0$ gives

$$
z_{0}=\omega_{0}+\lambda^{2} \int_{0}^{\infty} \frac{|f(\omega)|^{2}}{z_{0}-\omega} \mathrm{d} \omega
$$

Since the integral is always negative, $z_{0}$ is always smaller than $\omega_{0}$ which means the bound state must move down from the original one. In our specific model, the bound state generated from the discrete state on the first sheet will always move down towards negative infinity. For small $\lambda$, on the second sheet, besides the virtual pole originating from the pole of the form factor, there is another virtual state pole which is also generated from the original discrete state. We will give a general argument of the existence of this virtual pole in the next section. These two virtual poles come together as $\lambda$ increases and merge to be a second-order pole. As in previous case, at the merging point, we set the three solutions to be $u_{1,2}=-i d$ and $u_{3}=-i d_{0}$, and then Eq. (25) can also be used here to describe the merging point. From (25), $d$ can be positive or negative. The negative one corresponds to an imaginary $\lambda$ which is not physical. So, the two virtual-state poles can only merge once and then separately move onto the complex plane and become a pair of resonance poles. (see Fig. 5 ).

\section{FURTHER DISCUSSION ABOUT THE EXISTENCE OF THE VIRTUAL STATES}

We have seen that there could be second-sheet virtual-state poles generated from the form factor or from the discrete states. In this section we will give a general argument for these states to exist.

First we look at the virtual states generated from the form factor. It is a general result that whenever there is a simple pole of the form factor, there is a second sheet zero point of $\eta(\omega)$ near this position as the coupling constant is turned on. In [12], the author noticed this phenomenon in two special examples, but they did not provide a general reasoning for this to happen. Here, we provide a general argument as follows. Suppose the pole of the form factor is at $\tilde{\omega}$ with $\tilde{\omega} \neq \omega_{0}$. The integral term in $\eta(\omega)$ in (9) is analytic on the first sheet but has a pole for $\eta(\omega)$ on the second sheet at the pole position of the form factor. This pole appears because the analytic continuation of $\omega$ to the second sheet to $\tilde{\omega}$ causes the integration contour to be deformed and pinched by the two poles in the integrand as shown in Fig. 6. Then, $\eta$ on the second sheet can be expressed as the first sheet $\eta$ plus a residue

$$
\eta^{I I}(\omega)=\eta^{I}(\omega)+2 \pi i \lambda^{2} G^{I I}(\omega)=\eta^{I}(\omega)-2 \lambda^{2} \pi i G(\omega),
$$

where the superscripts $I$ and $I I$ denote the first sheet and the second sheet, respectively. The last equation can be deduced using the Schwartz reflective relation for the real analytic function [25] and can also be understood as the analytic continuation of $\eta(\omega)$ from the lower rim of the cut up to the second sheet. In our previous model,

$$
\eta^{I I}(\omega)=\eta^{I}(\omega)-2 \lambda^{2} \pi i \frac{\sqrt{\omega}}{\omega+\rho^{2}} .
$$

Since $\eta^{I}(\omega)$ is regular at the $\tilde{\omega}$, the second term gives the pole term at $\tilde{\omega}$ to $\eta^{I I}$ which is the pinch singularity. Near this second-sheet pole of $\eta, \eta^{I I}(\omega)=0$ is of the form

$$
\frac{\lambda^{2} c_{1}(\omega)}{\omega-\tilde{\omega}}=\omega-\omega_{0} \Rightarrow\left(\omega-\omega_{0}\right)(\omega-\tilde{\omega})=\lambda^{2} c_{1}(\omega)
$$




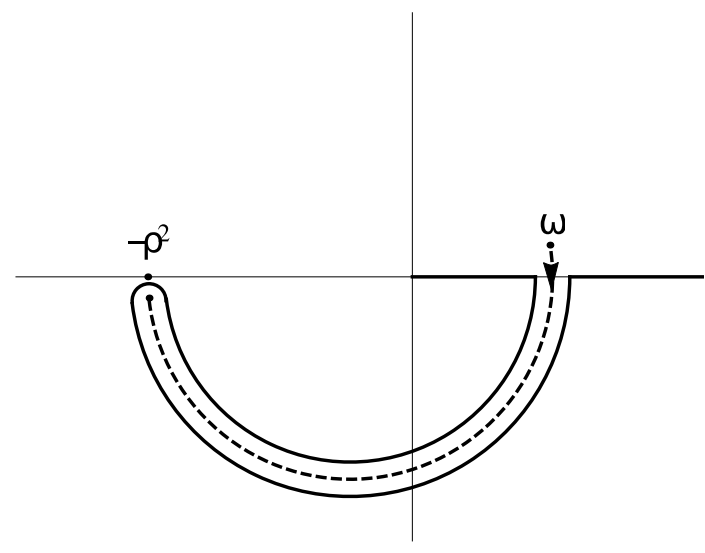

FIG. 6. The integral contour is pinched by the two poles as the $\omega$ analytically continued to the pole position of the form factor from the first sheet to the second sheet.

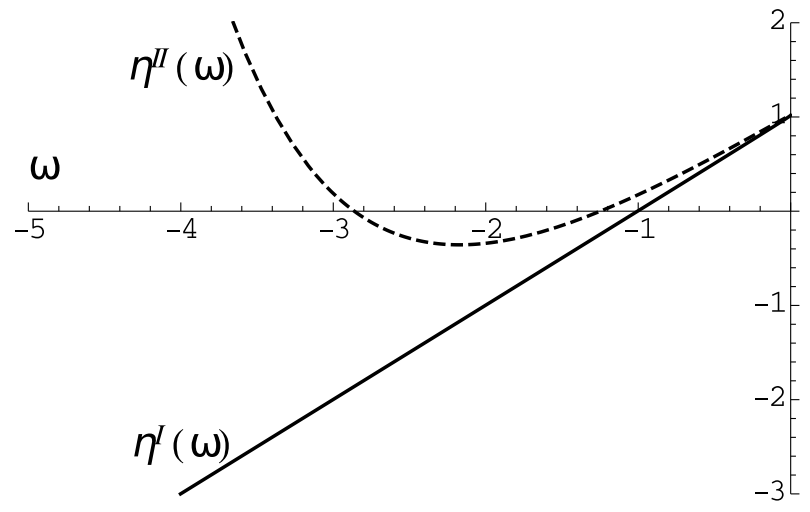

FIG. 7. $\eta(\omega)$ function on the first sheet and second sheet with form factor $G=\sqrt{\omega} e^{-\omega}, \omega_{0}=-1$, and $\lambda=0.1$.

where $c_{1}(\omega)$ is a function regular at $\tilde{\omega}$, and if $\tilde{\omega}$ is real and below the threshold, $c_{1}$ is real. At $\lambda=0$, only $\omega_{0}$ is the solution for the left equation. However, as $\lambda$ is turned on, the zero point originating from $\tilde{\omega}$ appears as illustrated in the left graph in Fig. 8 We can expand the solution with respect to $\lambda^{2}$. Suppose the zero point of $\eta^{I I}$ is at $\omega=\tilde{\omega}+\lambda^{2} \omega_{2}+O\left(\lambda^{4}\right)$, and above equation can be recast as

$$
-\left(\tilde{\omega}-\omega_{0}\right) \lambda^{2} \omega_{2}+O\left(\lambda^{4}\right)=\lambda^{2} c_{1}(\tilde{\omega})+O\left(\lambda^{4}\right) \Rightarrow \omega_{2}=-\frac{c_{1}(\tilde{\omega})}{\tilde{\omega}-\omega_{0}} .
$$

Thus, whenever the form factor has a pole, whether on the negative real axis or on the complex plane, there will be a state generated near the pole as the coupling is turned on.

In fact, a similar argument also applies to form factors such as $G(\omega)=\sqrt{\omega} e^{-\omega}$. In this case,

$$
\begin{aligned}
\eta^{I I}(\omega) & =\eta^{I}(\omega)-2 \pi i \lambda^{2} G(\omega)=\omega-\omega_{0}-\lambda^{2} \int_{0}^{\infty} d x \frac{G(x)}{(\omega-x)}-2 \lambda^{2} \pi i \sqrt{\omega} e^{-\omega} . \\
& =\omega-\omega_{0}+\lambda^{2} \int_{0}^{\infty} d x \frac{G(x)}{(-\omega-x)}+2 \lambda^{2} \pi \sqrt{-\omega} e^{-\omega} .
\end{aligned}
$$

in which the integral in the last line is real and goes to zero as $\omega \rightarrow-\infty$, while the last term goes to infinity at this limit. At exactly $\lambda=0, \eta(\omega)=\omega-\omega_{0}$ and goes to $-\infty$ as $\omega \rightarrow-\infty$. As long as $\lambda$ is turned on, $\eta^{I I}$ tends to $\infty$ as $\omega \rightarrow-\infty$. So for small enough $\lambda$, there must be one solution at large negative $\omega$ for the continuity of the function. See Fig. 7 for an illustration. As $\lambda$ is turned on gradually, this solution comes from negative infinity. From this example we conclude that if the form factor goes to $\infty$ as $\omega \rightarrow-\infty$ faster than $-\omega$ without other singularities, there will be a virtual state generated from the form factor from negative infinity as the coupling is turned on.

Next we look at the virtual state generated from the discrete state. In fact, this is also a general result. In general, whenever there is a discrete state coupled to a continuum below the threshold, as soon as the coupling is turned on, 

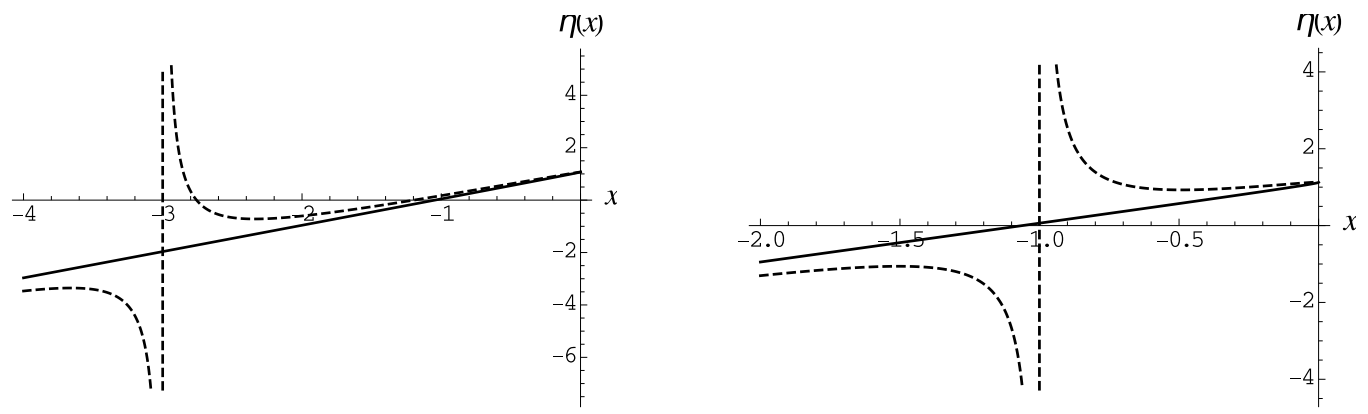

FIG. 8. Solid line and dashed line denote $\eta(x)$ on the real axis of the first and the second Riemann sheets, respectively. The left one : $\lambda=0.2, \rho^{2}=3$ and $\omega_{0}=-1$, with two virtual states; The right one $\lambda=0.2, \rho^{2}=1$ and $\omega_{0}=-1$, with a pair of resonance poles.

the original discrete state will be copied on both the first sheet and the second sheet and be renormalized separately. This is because before turning on the coupling $\eta(z)=z-\omega_{0},\left(\omega_{0}<0\right.$ and $\left.\omega_{0} \neq \tilde{\omega}\right)$, and as we turn on the coupling, on both Riemann sheets, $\eta(z)$ has small real corrections on the negative real axis near $\omega_{0}$, and the intersection between $\eta(z)$ and the negative axis should also move away continuously from $\omega_{0}$ on both sheets. See also the left figure in Fig. 8 for illustration. We can also expand the solution around $\omega_{0}$ with respect to $\lambda, \omega=\omega_{0}+\lambda^{2} \omega_{2}+O\left(\lambda^{4}\right)$, and $\eta^{I I}=0$ can also be expressed as

$$
0=\omega-\omega_{0}+\lambda^{2} c_{2}(\omega)=\lambda^{2} \omega_{2}+\lambda^{2} c_{2}\left(\omega_{0}\right)+O\left(\lambda^{4}\right) \Rightarrow \omega_{2}=-c_{2}\left(\omega_{0}\right)
$$

where $c_{2}(\omega)$ is regular at $\omega_{0}$. Perturbation theory in quantum theory only concerns the bound states, and the virtual state can only be reproduced by summing up the bubble chain and solving the inverse propagator on the second Riemann sheet. One exception is when the pole position of the form factor is the same as the energy for the discrete state $\omega_{0}$, and $c_{1}\left(\omega_{0}\right)<0$, where $c_{1}$ is defined in (29). The $\eta$ function is illustrated in the right figure of Fig.8, In this accidental case, there are no solutions on the negative axis but they will move to the complex plane and represent a pair of resonance poles of the $S$ matrix as soon as $\lambda$ is turned on. This can be seen from (29), which becomes

$$
\left(\omega-\omega_{0}\right)^{2}=\lambda^{2} c_{1}(\omega) .
$$

For small enough $\lambda$, we expand the solution with respect to $\lambda$ as before, $\omega=\omega_{0}+\lambda \omega_{1}+O\left(\lambda^{2}\right)$, and have

$$
\lambda^{2} \omega_{1}^{2}+O\left(\lambda^{3}\right)=\lambda^{2} c_{1}\left(\omega_{0}\right)+O\left(\lambda^{3}\right)
$$

and for $c_{1}\left(\omega_{0}\right)<0$, there can only be complex solutions.

\section{COMPLETENESS RELATION}

We have seen that besides the bound state and the continuum states, there are also resonant states and virtual states on the second sheet of the Riemann sheet. When the discrete state becomes resonance pole on the second sheet, only the continuum states form the complete set of bases and the completeness relation can be expressed using the states defined in terms of (10) as

$$
\mathbf{1}=\int_{0}^{\infty} d \omega\left|\Psi_{+}(\omega)\right\rangle\left\langle\Psi_{+}(\omega)\right| .
$$

Nevertheless, in Ref. 14], in order to solve the large Poincaré problem, Petrosky, Prigogine, and Tasaki (PPT) proposed to modify the continuum a little and then resonances can also appear in the completeness relation. In the spirit of PPT, the continuum states should be considered as a complex functional with integral contour information encoded in it. Two physical conditions, i.e., the decay of unstable state in the future and the emission of the outgoing wave, determine the rule for choosing the integral contour. In their derivation for the $\left|\Psi_{+}\right\rangle$state, the physical condition requires the pole position of the original discrete state to have a small $i \epsilon$ above the path on the real axis, and after the turning on of the interaction, the pole will move continuously down to the second sheet. The integral path for the continuum state should also be continuously deformed to keep the discrete state above the integration 


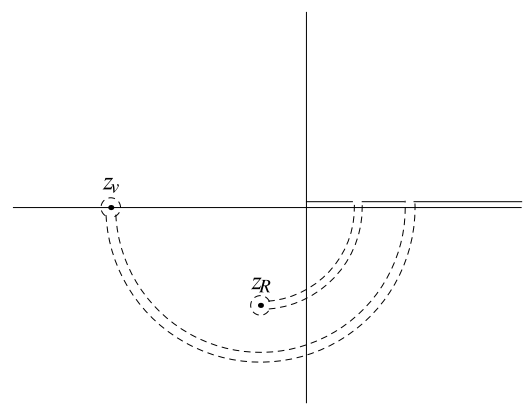

FIG. 9. The contour for $\eta_{d}^{+}$in the case with one virtual pole and a pair of resonance poles.

path as in Fig 1 Then one needs to include this path information into the continuum state by using $\eta_{d}^{+}$, which can also be expressed as

$$
\frac{1}{\eta_{d}^{+}(x)} \equiv \frac{1}{\eta^{+}(x)} \frac{x-\tilde{\omega}_{1}+i \gamma}{\left[x-\tilde{\omega}_{1}+i \gamma\right]_{+}}
$$

in which $\tilde{\omega}_{1}-i \gamma$ is the zero point of $\eta^{+}$and $[\ldots]_{+}$means deforming the contour as shown in Fig. 1] Similarly, for $\left|\Psi_{-}\right\rangle, \omega_{0}$ is taken to be a little below the real axis and $\eta^{-}$develops a zero above the real axis on the second sheet. Then the contour should be deformed upward into the second sheet to keep the pole below the contour, and one can include this contour information in $\eta_{d}^{-}=\frac{1}{\eta^{-}(x)} \frac{x-\tilde{\omega}_{1}-i \gamma}{\left[x-\tilde{\omega}_{1}-i \gamma\right]_{-}}$, where $[\ldots]_{-}$just denotes the deformation of the contour from the lower rim of the real axis to upper the second sheet. Now, the right continuum state can be expressed as $\omega>0$,

$$
\left|\Psi_{ \pm}(x)\right\rangle=|x\rangle+\lambda \frac{f(x)}{\eta_{d}^{ \pm}(x)}\left[|1\rangle+\lambda \int_{0}^{\infty} \mathrm{d} \omega \frac{f(\omega)}{x-\omega \pm i \epsilon}|\omega\rangle\right] .
$$

According to PPT's prescription, the left eigenstate with the same eigenvalue would not need the deformation of the contour,

$$
\left\langle\tilde{\Psi}_{ \pm}(x)\right|=\langle x|+\lambda \frac{f(x)}{\eta^{\mp}(x)}\left[\langle 1|+\lambda \int_{0}^{\infty} \mathrm{d} \omega \frac{f(\omega)}{x-\omega \mp i \epsilon}\langle\omega|\right] .
$$

The orthogonal relation for continuum states, $\left\langle\tilde{\Psi}_{ \pm}\left(x^{\prime}\right) \mid \Psi_{ \pm}(x)\right\rangle=\delta\left(x-x^{\prime}\right)$, still holds. With these definitions, the completeness relation can also be expressed in terms of the continuum and the resonant state,

$$
\mathbf{1}=\int_{0}^{\infty} d \omega\left|\Psi_{+}(\omega)\right\rangle\left\langle\tilde{\Psi}_{+}(\omega)|+| z_{R}\right\rangle\left\langle\tilde{z}_{R}\right|
$$

We can generalize these kinds of definitions to the cases with virtual states. Since the virtual states and resonances may transform to each other as the coupling changes, one should not treat them differently. So, whenever there are virtual states, it should also modify the integral path for the continuum states, and in the definition of $\eta_{d}^{ \pm}$in Eq. (37), all the second-sheet poles on the real axis and the lower half-plane should be included, which means the integral path is chosen as in Fig. 9. With these definitions of the continuum, the generalized completeness relation can be generalized here to Case 1, including the virtual state from the form factor

$$
\mathbf{1}=\int_{0}^{\infty} d \omega\left|\Psi_{+}(\omega)\right\rangle\left\langle\tilde{\Psi}_{+}(\omega)|+| z_{R}\right\rangle\left\langle\tilde{z}_{R}|+| z_{v}^{+}\right\rangle\left\langle\tilde{z}_{v}^{+}\right| .
$$

In Case 3, where the discrete state becomes a bound state and a virtual state for small $\lambda$, the generalized completeness relation should also include the two virtual states

$$
\mathbf{1}=\int_{0}^{\infty} d \omega\left|\Psi_{+}(\omega)\right\rangle\left\langle\tilde{\Psi}_{+}(\omega)\left|+\sum_{i=1}^{2}\right| z_{v i}^{+}\right\rangle\left\langle\tilde{z}_{v i}^{+}\right| .
$$

In general, as long as the contour for the continuum states goes around all the virtual-state poles and the resonance poles on the lower second sheet, these states also enter into the generalized completeness relation along with the bound states on the first sheet. 
Above completeness relations only apply to simple poles. We have also seen that there are second-order poles or third-order poles in our example. One would wonder what role these states play in the completeness relation. In fact, for simplicity, if there is only one second-order virtual-state pole and no other resonance or bound state, the generalized completeness relation is

$$
\begin{aligned}
\mathbf{1} & =\int_{0}^{\infty} d \omega\left|\Psi_{+}(\omega)\right\rangle\left\langle\tilde{\Psi}_{+}(\omega)|+| z_{v}^{+}\right\rangle\left\langle\tilde{z}_{v 2}^{+}|+| z_{v 2}^{+}\right\rangle\left\langle\tilde{z}_{v}^{+}\right|, \\
& =\int_{0}^{\infty} d \omega\left|\Psi_{-}(\omega)\right\rangle\left\langle\tilde{\Psi}_{-}(\omega)|+| z_{v}^{-}\right\rangle\left\langle\tilde{z}_{v 2}^{-}|+| z_{v 2}^{-}\right\rangle\left\langle\tilde{z}_{v}^{-}\right| .
\end{aligned}
$$

This can also be applied to the cases with complex double resonance poles on the complex plane by changing the virtual states to the corresponding resonant states. If there are more resonances or bound states, they must also included in the sum as above. Similar completeness relation in a special potential model in a different context was also found in [26].

This completeness relation can be generalized to the $n$ th-order virtual-state pole or resonance pole. In these cases, there are $n$ groups of Gamow states for an $n$ th-order pole, which can be represented as [20]

$$
\begin{aligned}
\left|z^{(1)}\right\rangle & =N\left(|1\rangle+\lambda \int_{0}^{\infty} \frac{f(\omega)}{[z-\omega]_{+}}|\omega\rangle \mathrm{d} \omega\right), \\
\left\langle\tilde{z}^{(1)}\right| & =N\left(\langle 1|+\lambda \int_{0}^{\infty} \frac{f(\omega)}{[z-\omega]_{+}}\langle\omega| \mathrm{d} \omega\right), \\
\left|z^{(n)}\right\rangle & =N(-1)^{n-1} \lambda \int_{0}^{\infty} \mathrm{d} \omega \frac{f(\omega)}{\left([z-\omega]_{+}\right)^{n}}|\omega\rangle, \quad \text { for } n \geq 2, \\
\left\langle\tilde{z}^{(n)}\right| & =N(-1)^{n-1} \lambda \int_{0}^{\infty} \mathrm{d} \omega \frac{f(\omega)}{\left([z-\omega]_{+}\right)^{n}}\langle\omega|, \quad \text { for } n \geq 2,
\end{aligned}
$$

$N=\left(\frac{n !}{\eta^{(n)}(z)}\right)^{1 / 2}=\left((-)^{n-1} \frac{\lambda^{2}}{n !} \int d \omega \frac{|f(\omega)|^{2}}{([z-\omega]+)^{n+1}}\right)^{-1 / 2}$ is chosen such that $\left\langle\tilde{z}^{(r)} \mid z^{(n-r+1)}\right\rangle=1$ and $z$ is the pole position either for resonances or virtual states. These are the states continued from the upper first sheet to the lower second sheet and there are also the other set of states continued from the lower first sheet to the upper second sheet. Suppose there are no other poles except the $n$ th-order pole, the completeness relation can be expressed as

$$
\mathbf{1}=\int_{0}^{\infty} d \omega\left|\Psi_{+}(\omega)\right\rangle\left\langle\tilde{\Psi}_{+}(\omega)\left|+\sum_{r=1}^{n}\right| z^{(r)}\right\rangle\left\langle\tilde{z}^{(n-r+1)}\right|,
$$

which is proved in the Appendix. In general, if there is more than one pole on the lower half-plane and the negative axis of the second sheet at positions $z_{j},\left(j=1, \ldots, m_{I I},\right)$ and also other simple bound-state poles $z_{b i},\left(i=1 \ldots, m_{b}\right)$ on the negative real axis of the first sheet, with the continuum states modified accordingly, the most general completeness relation can be written down as

$$
\mathbf{1}=\sum_{i}^{m_{b}}\left|z_{b i}\right\rangle\left\langle z_{b i}\left|+\int_{0}^{\infty} d \omega\right| \Psi_{+}(\omega)\right\rangle\left\langle\tilde{\Psi}_{+}(\omega)\left|+\sum_{j}^{m_{I I}} \sum_{r=1}^{n_{j}}\right| z_{j}^{(r)}\right\rangle\left\langle\tilde{z}_{j}^{\left(n_{j}-r+1\right)}\right|,
$$

where $n_{j}$ is the order of the $j$ th pole.

\section{CONCLUSION AND DISCUSSION}

In this paper, we first thoroughly studied the Friedrichs model with an integrable example form factor. As discovered in [12], the form factor introduces extra second-sheet poles besides the resonance poles originating from the discrete state. We give a general argument that each pole of the form factor may introduce an extra state on the second sheet. When the discrete state is below the threshold, besides the bound state, there is also a virtual-state pole arising from the discrete state for small coupling. We also give a general argument for the existence of such a virtual-state pole. This pole is similar to the shadow pole discussed in [13] in S-matrix theory. We also generalize PPT's prescription for the continuum states and give the completeness relations including all the states on the second Riemann sheet, i.e. all the virtual states and resonant states arise both from the discrete states and from the form factor. We also find that for larger coupling, the resonances that arise from the discrete state can meet on the negative real axis to 
form a double pole or meet with the other virtual state to form a triple pole. We have also discussed the generalized completeness relations including these higher-order resonances.

Note that in all the cases of the example, we have seen that the original discrete state is doubled as soon as the coupling is turned on. In Case 1, when $\omega_{0}>0$, it becomes a pair of resonance poles and in Case 3 when $\omega_{0}<0$, it becomes a bound state and a virtual state. In general, the number of the poles arising from the discrete state is always doubled whenever a new threshold is opened. The poles on different sheets with the same origin are called shadow poles by Eden and Taylor [13]. However, the number of the poles arising from the form factor is not doubled when the first threshold is opened. This is the difference between these two kinds of poles. The poles from the form factors can be regarded as dynamically generated states. In fact, in the dispersive analysis of the low-lying $0^{+}$resonances, $\sigma, \kappa$ are found to be just this kind of resonance [5]. In this paper, we only studied one opened channel. The number of the poles from the discrete state is only doubled once. When more channels are included, the number of the poles may be doubled more than once. In this sense, one original discrete state may generate more resonant states to be observed in the experiment. This phenomenon may already be observed in the low-energy $0^{+}$resonances [5].

\section{ACKNOWLEDGMENTS}

This work is supported by the China National Natural Science Foundation under Contracts No. 11105138, No. 11575177 and No. 11235010. Z.Z. also would like to thank the Project Sponsored by the Scientific Research Foundation for the Returned Overseas Chinese Scholars, State Education Ministry.

\section{Appendix A: Proof of the completeness relation for higher-order poles}

We assume that there is only one $n$ th-order pole $(n \geq 2)$ on the lower second sheet. The completion relation is

$$
\mathbf{1}=\int_{0}^{\infty} d \omega\left|\Psi_{+}(\omega)\right\rangle\left\langle\tilde{\Psi}_{+}(\omega)\left|+\sum_{r=1}^{n}\right| z^{(r)}\right\rangle\left\langle\tilde{z}^{(n-r+1)}\right| .
$$

Proof:

$$
\begin{aligned}
\int d \omega|\Psi(\omega)\rangle\langle\tilde{\Psi}(\omega)|= & \int|\omega\rangle\langle\omega|+\int d \omega \frac{\lambda^{2}|f(\omega)|^{2}}{\eta_{d}^{+}(\omega) \eta(\omega)}\left[|1\rangle\left\langle 1\left|+\int_{0}^{\infty} d \omega^{\prime} \frac{\lambda f\left(\omega^{\prime}\right)}{\omega-\omega^{\prime}+i \epsilon}\right| \omega^{\prime}\right\rangle\langle 1|\right. \\
& \left.+\int_{0}^{\infty} d \omega^{\prime} \frac{\lambda f\left(\omega^{\prime}\right)}{\omega-\omega^{\prime}-i \epsilon}|1\rangle\left\langle\omega^{\prime}\left|+\int_{0}^{\infty} d \omega^{\prime} d \omega^{\prime \prime} \frac{\lambda^{2}\left|f\left(\omega^{\prime}\right)\right|^{2}}{\left(\omega-\omega^{\prime}-i \epsilon\right)\left(\omega-\omega^{\prime \prime}-i \epsilon\right)}\right| \omega^{\prime}\right\rangle\left\langle\omega^{\prime \prime}\right|\right] \\
& +\int_{0}^{\infty} d \omega \frac{\lambda f(\omega)}{\eta^{-}(\omega)}|\omega\rangle\left\langle 1\left|+\int_{0}^{\infty} d \omega \frac{\lambda f(\omega)}{\eta_{d}^{+}(\omega)}\right| 1\right\rangle\langle\omega| \\
& +\int_{0}^{\infty} d \omega \frac{\lambda f(\omega)}{\eta^{-}(\omega)} \int_{0}^{\infty} d \omega^{\prime} \frac{\lambda f\left(\omega^{\prime}\right)}{\omega-\omega^{\prime}-i \epsilon}|\omega\rangle\left\langle\omega^{\prime}\right| \\
& +\int_{0}^{\infty} d \omega \frac{\lambda f(\omega)}{\eta_{d}^{+}(\omega)} \int_{0}^{\infty} d \omega^{\prime} \frac{\lambda f\left(\omega^{\prime}\right)}{\omega-\omega^{\prime}+i \epsilon}\left|\omega^{\prime}\right\rangle\langle\omega|
\end{aligned}
$$

Similar to the derivation in the Appendix of [14], the integrals above can be worked out as follows:

$$
\begin{aligned}
I_{1} & =\int d \omega \frac{\lambda^{2}|f(\omega)|^{2}}{\eta_{d}^{+}(\omega) \eta^{-}(\omega)}=-\left.\operatorname{Res} \frac{1}{\eta(\omega)}\right|_{z} ^{+}+1=1, \\
I_{2}\left(\omega^{\prime}\right) & =\int d \omega \frac{\lambda^{2}|f(\omega)|^{2}}{\eta_{d}^{+}(\omega) \eta^{-}(\omega)\left(\omega-\omega^{\prime}+i \epsilon\right)}=-\frac{1}{\eta^{-}\left(\omega^{\prime}\right)}+\frac{(-1)^{n} n !}{\eta^{+(n)}(z)\left[(z-\omega)_{+}\right]^{n}}, \\
I_{3}\left(\omega^{\prime}\right) & =\int d \omega \frac{\lambda^{2}|f(\omega)|^{2}}{\eta_{d}^{+}(\omega) \eta^{-}(\omega)\left(\omega-\omega^{\prime}-i \epsilon\right)}=-\frac{1}{\eta_{d}^{+}\left(\omega^{\prime}\right)}+\frac{(-1)^{n} n !}{\eta^{+(n)}(z)\left[(z-\omega)_{+}\right]^{n}}, \\
I_{4}\left(\omega^{\prime}, \omega^{\prime \prime}\right) & =\int d \omega \frac{\lambda^{2}|f(\omega)|^{2}}{\eta_{d}^{+}(\omega) \eta^{-}(\omega)\left(\omega-\omega^{\prime}+i \epsilon\right)\left(\omega-\omega^{\prime}-i \epsilon\right)} \\
& =\frac{1}{\omega^{\prime}-\omega^{\prime \prime}-i \epsilon}\left[-\frac{1}{\eta^{-}\left(\omega^{\prime}\right)}-\frac{1}{\eta_{d}^{+}\left(\omega^{\prime \prime}\right)}\right]+\frac{(-1)^{n} n !}{\eta^{+(n)}(z)} \sum_{r=0}^{n-1} \frac{1}{\left[(z-\omega)_{+}\right]^{r+1}\left[(z-\omega)_{+}\right]^{n-r}} .
\end{aligned}
$$


Inserting the above equations in (A2), the terms corresponding to the first terms in (A4), A5), and (A7) cancel the last three lines in $\mathrm{A} 2$ and we have

$$
\begin{aligned}
\int d \omega|\Psi(\omega)\rangle\langle\tilde{\Psi}(\omega)|= & \int|\omega\rangle\langle\omega|+| 1\rangle\langle 1| \\
& +\frac{(-1)^{n} n !}{\eta^{+(n)}(z)}\left[\int_{0}^{\infty} d \omega^{\prime} \frac{\lambda f\left(\omega^{\prime}\right)}{\left[\left(z-\omega^{\prime}\right)_{+}\right]^{n}}\left|\omega^{\prime}\right\rangle\left\langle 1\left|+\int_{0}^{\infty} d \omega^{\prime} \frac{\lambda f\left(\omega^{\prime}\right)}{\left[\left(z-\omega^{\prime}\right)_{+}\right]^{n}}\right| 1\right\rangle\left\langle\omega^{\prime}\right|\right. \\
& \left.+\sum_{r=0}^{n-1} \int_{0}^{\infty} d \omega^{\prime} \frac{\lambda^{2} f\left(\omega^{\prime}\right) f\left(\omega^{\prime \prime}\right)}{\left[\left(z-\omega^{\prime}\right)_{+}\right]^{r+1}\left[\left(z-\omega^{\prime \prime}\right)_{+}\right]^{n-r}}\left|\omega^{\prime}\right\rangle\left\langle\omega^{\prime \prime}\right|\right] \\
= & \mathbf{1}-\left[\left|z^{(n)}\right\rangle\langle 1|+| 1\rangle\left\langle\tilde{z}^{(n)}\left|+\int_{0}^{\infty} d \omega^{\prime} \frac{\lambda f\left(\omega^{\prime}\right)}{\left(z-\omega^{\prime}\right)_{+}}\right| \omega^{\prime}\right\rangle\left\langle\tilde{z}^{(n)}\right|\right. \\
& \left.+\int_{0}^{\infty} d \omega^{\prime} \frac{\lambda f\left(\omega^{\prime}\right)}{\left(z-\omega^{\prime}\right)_{+}}\left|z^{(n)}\right\rangle\left\langle\omega^{\prime}\left|+\sum_{r=1}^{n-2}\right| z^{(r+1)}\right\rangle\left\langle\tilde{z}^{(n-r)}\right|\right] \\
= & \mathbf{1}-\sum_{r=1}^{n}\left|z^{(r)}\right\rangle\left\langle\tilde{z}^{(n-r+1)}\right|
\end{aligned}
$$

and the completeness relation.

[1] K. A. Olive et al. (Particle Data Group), Chin. Phys., C 38, 090001 (2014).

[2] S. Godfrey and N. Isgur, Phys. Rev., D 32, 189 (1985).

[3] Z. Xiao and H. Q. Zheng, Nucl. Phys., A695, 273 (2001), arXiv:hep-ph/0011260 [hep-ph].

[4] H. Q. Zheng, Z. Y. Zhou, G. Y. Qin, Z. Xiao, J. J. Wang, and N. Wu, Nucl. Phys., A733, 235 (2004), arXiv:hep-ph/0310293 [hep-ph].

[5] Z.-Y. Zhou and Z. Xiao, Phys. Rev., D 83, 014010 (2011), arXiv:1007.2072 [hep-ph]

[6] E. Eichten, K. Gottfried, T. Kinoshita, K. D. Lane, and T.-M. Yan, Phys. Rev., D 17, 3090 (1978), [Erratum: Phys. Rev.D 21,313(1980)].

[7] Z.-Y. Zhou and Z. Xiao, Phys. Rev., D 84, 034023 (2011), arXiv:1105.6025 [hep-ph]

[8] Z.-Y. Zhou and Z. Xiao, Eur. Phys. J., A 50, 165 (2014), arXiv:1309.1949 [hep-ph]

[9] A. Bohm, J. D. Dollard, and M. Gadella, Dirac Kets, Gamow Vectors and Gel'fand Triplets, Lecture Notes in Physics, Vol. 348 (Springer Berlin Heidelberg, 1989) ISBN 978-3-540-51916-4 (Print) 978-3-540-46859-2 (Online).

[10] O. Civitarese and M. Gadella, Commun. Pure Appl. Math., 396, 41 (2004).

[11] K. O. Friedrichs, Commun. Pure Appl. Math., 1, 361 (1948).

[12] A. K. Likhoded and G. P. Pronko, Int. J. Theor. Phys., 36, 2335 (1997).

[13] R. J. Eden and J. R. Taylor, Phys. Rev., 133, B1575 (1964).

[14] T. Petrosky, I. Prigogine, and S. Tasaki, Physica, 173A, 175 (1991).

15] N. Nakanishi, Prog. of Theor. Phys., 19, 607 (1958).

[16] T. Petrosky, G. Ordonez, and I. Prigogine, Phys. Rev., A62, 042106 (2000).

[17] G. Ordonez, T. Petrosky, and I. Prigogine, Phys. Rev., A63, 052106 (2001).

[18] M. Gadella, J. Math. Phys., 24, 2142 (1983).

[19] M. Gadella, J. Math. Phys., 25, 2481 (1984).

[20] I. E. Antoniou, M. Gadella, and G. P. Pronko, J. Math. Phys., 39, 2459 (1997).

[21] E. Hernandez, A. Jáuregui, and A. Mondragon, J. Phys. A, 33, 4507 (2000).

[22] I. Antoniou, M. Gadella, J. Mateo, and G. Pronko, Int. J. of Theor. Phys., 42, 2389 (2003).

[23] A. Mondragon and E. Hernandez, J. Phys. A, 26, 5595 (1993).

[24] A. Bohm, M. Loewe1, S. Maxson, P. Patuleanu1, C. Püntmann, and M. Gadella, J. Math. Phys., 38, 6072 (1997).

[25] For $F(z)$ real on the negative axis and complex on the positive real axis, $F(z)$ can be decomposed as $F(z)=R(z)+i G(z)$, $R(z)=\operatorname{Re} F(z), G(z)=\operatorname{Im} F(z)$, for $z \in \mathbb{R}_{+} \cdot R(z)$ and $G(z)$ are the analytic continuations of $\operatorname{Re} F(z), \operatorname{Im} F(z), \operatorname{respectively,}$ from the positive axis. From Schwartz reflection principle and the uniqueness of the analytic continuation, $F^{*}(z)=F\left(z^{*}\right)$, $R^{*}(z)=R\left(z^{*}\right), G^{*}(z)=-G\left(z^{*}\right)$ on the first sheet. However, the Schwartz reflection principle also requires that the continuation of $G(z)$ satisfies $G^{*}(z)=G^{I I}\left(z^{*}\right)$, which means $G(z)=-G^{I I}(z)$.

[26] E. Hernández, A. Jáuregui, and A. Mondragón, Int. J. Theor. Phys., 42, 2167 (2003). 